\title{
Escuelas de Medicina: los estudiantes de hoy
}

Departamento de Educación en Ciencias de la Salud; Facultad de Medicina; Universidad de Chile. aMagister en Educación en Ciencias de la Salud.

Recibido el 10 de septiembre de 2010 , aceptado el 18 de enero de 2011.

Correspondencia: Dra. Natasha Kunakov Facultad de Medicina, Universidad de Chile. Fono 9786002 E-mail:nkunakov@med. uchile.cl

\author{
NATASHA KUNAKOV
}

\section{Medical schools: Students today}

Physicians that are faculty members in medical schools receive new students every year, and they are expected to prepare those students to become professionals. They usually appeal to their experience to meet that challenge. However, newer generations of students are different, and experience, with no formal training for teaching them, can be insufficient. New characteristics of students can be related to their early contact in life with information technology. Their brain has been somehow modified by stimuli offered by this technology, and the way they learn has also been modified. This paper is a reflection about how students have changed and it analyzes how their learning experience needs to be modified accordingly. Teaching based only on experience might be insufficient to fulfill the expectations of young students that have chosen the medical profession for their future.

(Rev Med Chile 2011; 139: 524-528).

Key words: Faculty, medical; Schools, medical; Teaching.
"Para enseñar latín a Pedro, se debe saber latín, pero también se debe saber Pedro"

(Proverbio jesuita)

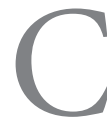

ada año los médicos que actuamos como docentes en las Escuelas de Medicina nos enfrentamos a desarrollar el programa de la asignatura para el próximo período académico. Lo habitual es que estas programaciones sean desarrolladas basándose en los conocimientos que deberían adquirir los estudiantes; comúnmente no tomamos en cuenta sus características. Sin embargo, para poder actuar acertadamente en el contexto educativo es necesario conocer cuál es la "materia prima" sobre la cual ejercemos nuestra acción.

Este artículo pretende acercarse a una caracterización de los estudiantes que ingresan a las Escuelas de Medicina, con el objetivo de entregar algunos elementos a los médicos docentes para hacer más exitosa su labor.

\section{La tecnología y cómo ha cambiado a los estudiantes}

Los estudiantes de hoy no sólo están usando la tecnología, sino que su manera de abordar la vida es diferente a la de generaciones anteriores debido a la misma tecnología. Marc Prensky ${ }^{1}$, escritor, consultor y diseñador de juegos para la educación y el aprendizaje, designó a esta generación como los "nativos digitales", porque son "nativos" del lenguaje digital de los juegos por computadora, video e Internet. Antes de entrar a la universidad han participado de 10.000 horas de video juego, 200.000 mails enviados y recibidos, 10.000 horas celular, 20.000 horas TV, 500.000 comerciales vistos, haciendo de estos elementos parte integral de sus vidas. Desde la misma perspectiva también se les ha llamado la "generación multimedia" "donde los jóvenes aprenden al mismo tiempo a utilizar un control remoto, un teléfono celular y un computador"

Esta generación tiene una forma muy diferente 
Escuelas de Medicina: Los estudiantes de hoy - N. Kunakov

de enfrentar el aprendizaje, piensan y procesan la información de manera distinta. Gustan de los procesos y multitareas paralelos, prefieren los gráficos antes que el texto, defienden los accesos al azar (desde hipertextos), funcionan mejor cuando trabajan en red, prosperan con satisfacción inmediata y bajo recompensas frecuentes. Usan los aparatos digitales en forma paralela (notebooks, ipod, celulares y otros). Se mueven en un universo de dinamismo de fragmentación, de inmediatez, un mundo mosaico, de continua estimulación, y donde todo es simultáneo. Como corolario: "Generación capaz de manejar información y tomar decisiones muy rápido con procesamiento paralelo". Para esta generación el "zapping" ha dejado de ser una actitud frente al televisor, se ha convertido en una actitud frente a la vida ${ }^{2}$.

\section{Una mirada desde la neurobiología}

Es conocido que el cerebro es un órgano que en el momento de nacer ha alcanzado el número total de células que persistirán hasta que se vayan irremediablemente destruyendo con el paso de la vida. Paralelamente, crece el número de dendritas y con ello de sinapsis, aumentando la interconexión con otras neuronas; produciéndose un proceso de arborización cada vez más complejo, y continúa durante toda la vida, es lo que se ha llamado la "plasticidad neuronal". Implica que todos nacemos y desarrollamos miles de millones de sinapsis, más de las que necesitamos; estas sinapsis representan las conexiones potenciales entre las neuronas que podrían necesitarse para crear mapas y modelos internos de todos los mundos posibles en los cuales pudiéramos movernos. Las conexiones que son usadas, al contrastarse con el mundo "real", se fortalecen y las que no se usan se pierden; este proceso se ha llamado "poda neuronal" ${ }^{3,4}$.

Por lo tanto, se puede afirmar que el desarrollo cerebral está influenciado y dirigido por condiciones ambientales, entendiendo el ambiente como todas las influencias externas, una lista infinita que abarcan los aspectos físicos del ambiente (nutrición, sonidos, cuidado, afecto, olores, aprendizaje) hasta la propia cultura, entendida ésta como las estructuras económicas, sociales y políticas, las formas de vida y de comportamiento ${ }^{4}$.

Aprender implica formar nuevas conexiones entre las neuronas y la elaboración de proteínas para que esto ocurra. Desde esa postura puede decirse que un ambiente enriquecido, lleno de estímulos externos y afectivos, aumenta el aprendizaje, es capaz de transformar un cerebro. La práctica activa los circuitos neuronales. Mientras más se practica, hay más síntesis de proteínas, se crean conexiones nerviosas y hay más aprendizaje. El estímulo que entrega un pizarrón sirve para cierto tipo de aprendizaje, deja muchas conexiones sin estímulo, por ello la enseñanza no puede quedar limitada al pizarrón; se deben buscar nuevas formas de captar la atención y la emoción del estudiante, hecho que es de vital importancia, ${ }^{3,5}$.

Desde la propuesta del modelo de la neurobiología, podemos decir que las diversas clases de experiencia conducen a estructuras cerebrales diferentes, lo cual tiene abundante evidencia en estudios globales 3,5 . "En investigaciones realizadas hay evidencia que las habilidades cognitivas (aprender a leer, a sumar, etc.) son moldeables hasta edades muy tempranas, a diferencia de las habilidades no cognitivas (ser responsable, puntual, etc). Es decir, si un niño no es estimulado intelectualmente desde pequeño, entonces es muy difícil y costoso que aprenda después, lo que no ocurre tan drásticamente cuando se trata de enseñar o cambiar comportamientos".

Por otra parte, considerando que los estudiantes chilenos están inmersos en este mundo globalizado y los estudios sociales realizados muestran que están sometidos a los mismos estímulos que se dan en otras latitudes, sus cerebros habrían cambiado como resultado de su formación y contacto con la tecnología ${ }^{7}$. De estas situaciones es plausible inferir que el contacto con la tecnología ha contribuido a que los patrones de pensamiento de los estudiantes que hoy ingresan a las Escuelas de Medicina sean distintos a los que se daban años atrás.

\section{Aclarando la paradoja “Más comunicaciones interneuronales, menos comunicación interpersonal?"}

A la luz de lo consignado pareciera que la tecnología hubiera influenciado negativamente la comunicación con los otros. Es decir, los jóvenes aparecen en solitario frente a su "computador personal", no observan al otro, no entran en diálogo directo, no usan la riqueza del lenguaje ni advierten las inflexiones de la voz, no tienen la 
posibilidad de observar la postura (situación que está cambiando a través del uso en tiempo real de cámaras web); la comunicación se realiza de manera escrita, pero no como intercambio epistolar, sino de corte utilitario, telegráfica, privilegiando la inmediatez, las palabras se transforman en símbolos, y pareciera no haber tiempo para la reflexión. En este contacto se pierde la comunicación no verbal que hasta ahora había formado parte indisoluble de la comunicación humana.

Estas observaciones son recogidas por algunos académicos quienes opinan que los nuevos estudiantes no validan o no perciben a los otros, centrándose en sí mismos. El escritor e historiador Jocelyn-Holt ${ }^{8}$ los describe como "individualistas, narcisistas, competitivos y agresivos", también comenta que los lleva a considerar con escepticismo o fría indiferencia el conocimiento o las habilidades que otros pueden enseñarles. Esta desvalorización del otro llevaría a una ausencia de modelos para superar situaciones frustrantes o difíciles.

Sin embargo, esta observación no se condecía con lo observado en las conexiones neuronales, ni con el hecho admitido que el ser humano es un ser social, lo cual motivó revisar los comportamientos de los adolescentes respecto del uso de las nuevas tecnologías. Estudios realizados en Estados Unidos de Norteamérica, Europa, México y Argentina muestran que la comunicación no ha desaparecido, sino que se ha transformado. Y los nuevos medios tecnológicos no están provocando una marginalización de los jóvenes, sino por el contrario, son el soporte para su sociabilidad ${ }^{2}$.

Podemos entender la cultura de una sociedad, incluida la cultura popular, como un "manual" que ayuda a un individuo a entender la sociedad y a vivir en ella. La cultura implica también la capacidad de estructurar y de relacionar los saberes con los que se cuenta. Si el capital cultural es débil, el patrimonio cultural social también lo será. Las nuevas tecnologías modifican este capital cultural, pero por sobretodo modifican la manera de adquirirlo.

Los cambios parecen iniciarse con la aparición de la "cultura de la casa" en los años 70, donde la presencia del televisor convoca a las familias en el hogar, hecho que se acentúa con el crecimiento urbano y la inseguridad de las calles, lo cual sacrifica la vida de los niños en los espacios públicos. Aquí aparecen las naturales diferencias entre los integrantes y se van creando espacios individuales, como la habitación, entendida como un "espacio propio", que se estructura y se vive como tal. Pero, el ser humano es implícitamente un "ser social" y los adolescentes permanecen en la máxima "para ser uno mismo entre los otros hay que ser y hacer como los otros", acuñada por Morduchowicz. El uso de las nuevas tecnologías por los adolescentes se aboca a cumplir el objetivo de socialización. Los estudios muestran que $78 \%$ de los jóvenes usan la computadora para hacer chat, 70\%, para jugar, $70 \%$ para hacer la tarea, $60 \%$ para buscar información; en este ranking parece ser más favorecida la sociabilización y la entretención ${ }^{2,7}$.

A pesar del uso social que dan los estudiantes a las nuevas tecnologías, la falta de integración de éstas en la educación no permite sacar partido a toda su potencialidad. Se dice que "vivir en una sociedad de la información y en una economía basada en el conocimiento requiere que sus jóvenes posean una amplia gama de competencias en este campo para que puedan participar plenamente como ciudadanos, ya que tales competencias se constituyen como componentes esenciales de la educación del siglo XXI" . Viendo esto puede decirse que el acceso ilimitado pero indiscriminado a la información a través de la WEB, puede llevarlos a una actitud enciclopedista superficial y sin integración del saber.

\section{El impacto de algunos cambios sociales}

La masificación de la universidad también influye en los cambios en estilos de aprendizaje que presentan los estudiantes. Debe considerarse que la capacidad de los estudiantes para ingresar a la universidad se mide por pruebas de aptitud, las cuales involucran una cantidad de conocimientos adquiridos y por ende, favorecen a los estudiantes de niveles socioeconómicos más elevados. Los cupos en las universidades se van llenando de acuerdo al ranking que tenga dicha universidad en el mercado, de ese modo, las universidades de mayor ranking tendrán estudiantes de mejor calidad académica ${ }^{10}$.

El aprendizaje de un estudiante es distinto si tuvo sobre estimulación precoz en su infancia, desarrolló una red neuronal más potente, a la de una persona que ha sido insuficientemente estimulada $^{4,5}$, pero que también entra a la educación 
superior a través de la oportunidad que le brinda la masificación de la universidad, y en este contexto, los programas de las Escuelas de Medicina deben tomar en consideración "al tipo de público que van a atender".

También se agrega que hoy día, hay acuerdo en la mala calidad que presentan los egresados de la educación media chilena, comentado ampliamente en seminarios de educación superior, situación que se repite a nivel mundial como lo expresa la UNESCO en sus documentos ${ }^{11,12}$. "Una de las falencias es la precaria enseñanza de competencias para manejarse en esta sociedad de la información", esto referido a que el sistema educativo no les entrena para un adecuado uso de la tecnología.

Respecto de los estudiantes que ingresan a medicina, se dice "Hoy en día, la elección de estudiar una carrera como la de Medicina se da más por ser vista como una profesión supuesta de élite y de atractivo social no cuestionado, y no tanto por vocación de servicio" ${ }^{13}$. En este contexto podemos decir que la motivación de ingreso a las carreras también se ve afectada por los cambios, Sin embargo, es conocido que los aspectos motivacionales son el motor más importante para lograr el proyecto individual, lo cual debe ser tenido en consideración a la hora de desarrollar los programas de aprendizaje en las Escuelas de Medicina.

Como resumen, el perfil académico de ingreso en algunas Escuelas de Medicina no es de los mejores, pero una vez que la institución les ha abierto sus puertas, les entrega el derecho a egresar como profesional. Los médicos docentes deben estar preparados para preocuparse de sus características y lograr que el estudiante sea exitoso; es la institución y los docentes quien los certifica como médicos ante la sociedad.

\section{¿Cómo se podría caracterizar a los estudiantes que están ingresando a las escuelas de medicina hoy?}

Considerando los antecedentes entregados podría decirse que los estudiantes que ingresan a las Escuelas de Medicina hoy día tendrían un alto grado de manejo de las tecnologías y de la hipertextualidad, serían capaces de manejar información y tomar decisiones muy rápido con procesamiento paralelo. Además, poseen motivaciones que los movilizan a adquirir los conocimientos necesarios y tendrían una actitud de escepticismo o de fría indiferencia respecto de los que los médicos docentes podrían aportarles. Presentarían una ausencia de modelos para superar situaciones frustrantes o difíciles, una actitud enciclopedista superficial y sin integración del saber; su relación sociedad-estudiante se caracterizaría por una tendencia a la desvinculación, la autodeterminación y la satisfacción de impulsos como el supremo bien.

Todo lo anterior indica que vendrían con una capacidad cognitiva "distinta", ante la cual el formato tradicional de la enseñanza estaría obsoleto, y se necesita que los médicos docentes trabajen nuevos estrategias para el desarrollo del aprendizaje.

\section{¿Y los médicos docentes? Sus contradicciones}

Un problema de difícil solución en la educación es que los médicos docentes actuales son considerados inmigrantes digitales, es decir, hablan una lengua anticuada y tratan de enseñar a una población que habla perfectamente esta nueva lengua. Los inmigrantes digitales no logran comprender que sus estudiantes pueden aprender con éxito mientras ven la TV o escuchan música, porque ellos no pueden y tienen poco aprecio por estas habilidades de los nativos.

La distinción entre medios nuevos y tradicionales no tiene sentido para los estudiantes. Son los médicos docentes los que perciben las rupturas tecnológicas, y, por tanto, es su función proyectar los nuevos aprendizajes a desarrollar y los nuevos usos sociales de las innovadoras tecnologías que deben utilizar. La postura más habitual de los médicos docentes es obviar el cambio, y considerar que los mismos métodos que usaron sus profesores cuando ellos eran estudiantes, servirán para sus estudiantes.

Desafortunadamente para los médicos docentes inmigrantes digitales, la gente que acude a sus clases creció a otra velocidad, utilizan instantáneamente el hipertexto, descargan música, telefonean desde aparatos de bolsillo, la biblioteca está en sus computadoras portátiles, emiten mensajes y chatean de manera inmediata. Trabajan en red toda su vida y tienen poca paciencia para las conferencias y la lógica paso a paso.

Para desarrollar una educación médica de ca- 
lidad, los médicos docentes deben prepararse para responder ante el nuevo escenario que implica este cambio en los estudiantes. Entonces la propuesta es realizar cambios, tanto en metodología como en contenidos, que respondan a estos nuevos atributos y necesidades. En principio, los médicos docentes tienen que aprender a comunicarse en el lenguaje y estilo de los estudiantes. Los avances en Educación Médica permiten enfrentar este paradigma ayudando en la habilitación docente, principalmente en lo referente metodologías de aprendizaje y de evaluación, demostradamente válidas y confiables para apoyar a esta nueva generación.

Pero esta pregunta es fundamental y trascendente, ¿Cuántos médicos docentes inmigrantes digitales, se reconocen en esta condición y quieren prepararse para enfrentar estos cambios en la Educación Médica?

\section{Referencias}

1. Prensky M. "Digital Natives Digital Immigrants, On the Horizon”. MCB University Press, MCB; vol 9, N⒌ 2001. Disponible en: www.marcprensky.com [consultado en octubre de 2010].

2. Morduchowicz R. "La generación Multimedia". Buenos Aires, Argentina: PAIDOS SAICF, 2008. Varios capítulos.

3. Solms M, Turnbull O. "El cerebro y el mundo interior". Mexico : Fondo de cultura económica, 2005. Capítulo
7 "Influencias genéticas y ambientales en el desarrollo mental".

4. Levi-Montalcini R. Tiempo de cambios. Barcelona : Ediciones Península, 2005. Varios capítulos.

5. Álvarez M. Datos blandos para ciencias duras. Buenos Aires, Argentina: PAIDOS SAICF, 2009. Capítulo 3 "Mecanismos mente-cuerpo".

6. Carneiro P, Heckman J. "HUMAN CAPITAL POLICY”. Chicago: National Bureau of Economic Research, 2003. Capítulo I "Introduction and motivation".

7. Instituto Nacional de la Juventud. Sexta encuesta nacional de la juventud. Santiago. INJUV, 2010. Capítulo XI "Uso de Tecnología".

8. Jocelyn-Holt A. “¿Somos autistas?” La Tercera; Ideas \& debates, 20 de enero de 2008.

9. Bacher S. Tatuados por los medios. Buenos Aires, Argentina: PAIDOS SACIF, 2009. Capítulo 11 "Construcción de estereotipos juveniles".

10. Brunner J, Uribe D. Mercados Universitarios: el nuevo escenario de la educación superior. Santiago: Universidad Diego Portales, 2007. Parte I, subsección: "La competencia por prestigios". Disponible en: www.brunner.cl. [Consultado en octubre de 2010].

11. UNESCO. World declaration on Higer education for the twenty-first century: Vision and Action. UNECO, 1998.

12. UNESCO. Final report of the Meeting of Higher Education Partners. UNESCO, 2004.

13. Rosselot E, Norero C, Hanne C. Formación profesional y su acreditación en medicina, un paradigma en garantía de la fe pública. Santiago. Rev Med Chile 2002; 130: 585 9. 